\title{
Unraveling the origin of exponential law in intra-urban human mobility
}

SUBJECT AREAS:

APPLIED PHYSICS

STATISTICAL PHYSICS

Received

19 July 2013

Accepted

27 September 2013

Published

18 October 2013

Correspondence and requests for materials should be addressed to

K.X. (kexu@nlsde. buaa.edu.cn)
Xiao Liang, Jichang Zhao, Li Dong \& Ke Xu

State Key Lab of Software Development Environment, Beihang University, Beijing 100191, P.R. China.

The vast majority of travel takes place within cities. Recently, new data has become available which allows for the discovery of urban mobility patterns which differ from established results about long distance travel. Specifically, the latest evidence increasingly points to exponential trip length distributions, contrary to the scaling laws observed on larger scales. In this paper, in order to explore the origin of the exponential law, we propose a new model which can predict individual flows in urban areas better. Based on the model, we explain the exponential law of intra-urban mobility as a result of the exponential decrease in average population density in urban areas. Indeed, both empirical and analytical results indicate that the trip length and the population density share the same exponential decaying rate.

nderstanding human movement patterns is considered as a long-term fundamental but challenging task for decades. It is an essential component in urban planning ${ }^{1-3}$, epidemics spreading ${ }^{4-6}$ and traffic engineering $^{7-9}$. With the mobile positioning technology (e.g., GPS, cellular towers and Wi-Fi) widely used in our daily lives over the years, massive amounts of individual tracks can be observed and recorded, which provides a great opportunity to the research of human mobility patterns.

In recent studies, one of the important discoveries is that the patterns of human movements in large scale of space, including trips between counties or cities, exhibit the Lévy walk characteristic ${ }^{10-13}$, which is also observed in animal motions ${ }^{14-17}$. For instance, the measured trip lengths can be well approximated by fat-tailed distributions through investigating the dispersal of bank notes in the United States ${ }^{10}$ and mobile phone records in European countries $^{11}$. While regarding to the human movements in urban areas, the latest research shows the scaling law is absent ${ }^{18-24}$. Be specific, several studies report exponential-tailed distributions of travel distances produced by various transportation means: taxis ${ }^{18-20}$, private $\operatorname{cars}^{21}$ and subways ${ }^{22}$. Furthermore, the exponential decaying of trip lengths is also demonstrated respectively in eight cities of Northeast China by analyzing the mobile call records ${ }^{23}$. It is worth noting that distinct characteristic trip lengths are observed in different cities ${ }^{23,24}$. In order to understand the origin of the scaling law, several possible explanations from the viewpoint of individual movements $^{27-29}$ are presented. The scaling law is also employed to model mobility patterns directly, which can reproduce some statistical features of human trajectories ${ }^{12}$. While with respect to intra-urban mobility, it is more necessary to explore its patterns, because for most citizens the majority of their trips occur in urban areas and just traverse small distances. Some studies conjecture that the exponential law is produced by trips from a single means of transportation and the scaling law is obtained by aggregated trips from all kinds of transportation ${ }^{25,26}$. But the exponential tails of trip lengths can also be observed in intra-urban human movements captured by mobile phone calls ${ }^{23}$ and location-based services ${ }^{24}$, which are not restricted to modes of transportation. Besides, the idea of intra-urban movements are driven by the distribution of points of interest (POIs) ${ }^{24}$ might be challenged by the fact that the visit probabilities of POIs depend not only on their geographical locations, but also on their sizes and popularities. To sum up, although more and more evidence demonstrates the exponential law in intra-urban human movements, the convincing origin of this universal rule is still missed.

Because most empirical studies aforementioned are based on collective human trips while there is no strong evidence that individual movements have the similar patterns with collective motions ${ }^{24,25}$. In this paper, we explore the statistical characteristics of intra-urban mobility and uncover the origin of the exponential law from the perspective of collective movements. A new model is presented to predict individual flows between different regions with high fidelity, which could also reproduce the actual distributions of trip lengths in cities. Then from this model, we find that the traffic flux depends on the spatial population distribution heavily. Finally, both the empirical simulations and the analytical proof indicate that the exponential law is caused by the distribution of population density and they also share the same decaying rate. Our results can help deepen the understanding of intra-urban movements further and provide important guidance to model individual movements in urban areas. Moreover, our findings can offer new insights into the origin of different laws at different space scales. 


\section{Results}

Modeling collective intra-urban mobility. In this paper, human travel records were collected from four great cities by taxis, subways and surveys (see Methods section). It is demonstrated that the exponential law of collective human movements does exist in urban areas of cities (see Supplementary Information (SI) section I).

In order to understand the exponential law of collective human mobility in urban areas, it is essential to model individual flows from one region to the other in a city. Although the gravity model ${ }^{30}$ has already been applied widely to predict flows, including human travel $^{4,8}$, cargo ship movement ${ }^{31}$ and telephone communications ${ }^{32}$, it still has some flaws such as incompetence to explain the discrepancy of the numbers of individual flows in both directions between a pair of locations. Then in order to fix its disadvantages, Simini et al. ${ }^{33}$ put forward the radiation model without parameters. In this model, the expected flux $\left\langle T_{i j}\right\rangle$ from location $i$ to $j$ is defined as

$$
\left\langle T_{i j}\right\rangle=T_{i} \frac{P_{i} P_{j}}{\left(P_{i}+P_{i j}\right)\left(P_{i}+P_{j}+P_{i j}\right)},
$$

where $P_{i}$ and $P_{j}$ are the populations of location $i$ and $j, T_{i}$ is the number of trips starting from $i$ and $P_{i j}$ is the total population of locations (except $i$ and $j$ ) from which to $i$ the distances are less than or equal to $d_{i j}$ (the distance between $i$ and $j$ ). The model can predict population movements between counties or cities successfully ${ }^{33}$, but it is not clear whether the model applies to intra-urban movements as well.

Especially noteworthy is that in urban areas, it is difficult to obtain population distribution directly because of high mobility. Moreover, because people often move frequently for various purposes in cities (e.g., go to work, visit their friends and go shopping), it is unsuitable to use resident population to model individual flows. Compared to the resident population, the average daily population occurring in a zone of city is more reasonable to characterize the urban mobility ${ }^{9}$, which is because it reflects routine travel behaviors and establishes a bond between human travel intensity and the function of the zone. So, in this paper, we regard the number of trips arriving at a zone as the population of the zone, which is proportional to the actual average daily population approximately.

Because the gravity model can not distinguish the directions of human flows, we only verify whether the radiation model is applied to predict human flows in urban areas. After calculating the population of zones, the results of simulation in Beijing by the radiation model is shown in Fig. 1. From the figure, it seems that the predicted flux has a large deviation from the actual ones and the model underestimates the probabilities of trips with distances larger than $1 \mathrm{~km}$.

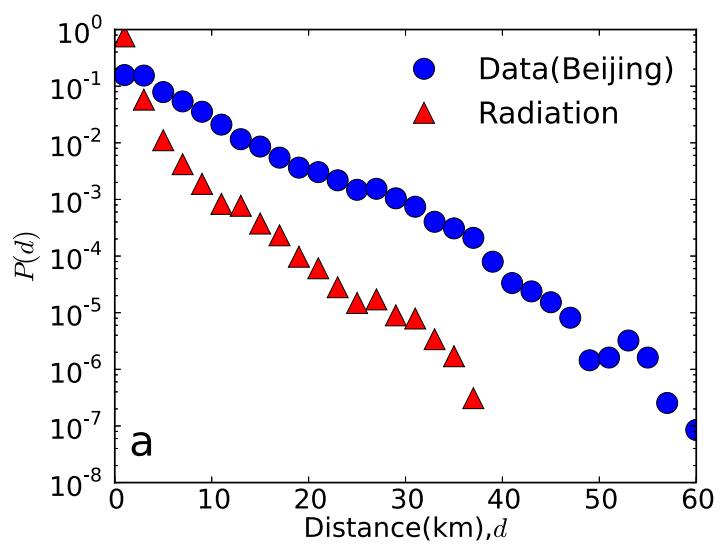

Similarly, the same phenomena can also be observed in other three cities, which are not included here. The possible reason to account for the incapability of radiation model is that there are different travel habits and preferences existing in trips at different scales of space. Therefore, it is necessary to consider a new model to understand intra-urban human mobility patterns.

Inspired by the gravity model ${ }^{30}$, we assume that the probability arriving at a location has a positive correlation with the population density of the location, but has a negative correlation with the Euclidean distance between the location and the originated location. Hence, in our model the probability of a trip reaching the location $n$, conditioned on starting from the location $m$, is defined as follows

$$
P(n \mid m) \propto \frac{\rho(n)}{f\left(d_{m n}\right)},
$$

where $\rho(\cdot)$ is the population density function and $f(d)$ is a function of distance between locations, which is usually given by two frequently used forms: power law and exponential.

Likewise, as for regions, the probability of a trip arriving at the region $j$, conditioned on originating from the region $i$, is defined as

$$
P(j \mid i) \propto \frac{P(j)}{f\left(d_{i j}\right)},
$$

where $P(\cdot)$ is the population of regions. Then the probability of a trip from region $i$ to $j$ can be derived as

$$
\begin{aligned}
P(i \rightarrow j) & =P_{\text {norm }}(i) P(j \mid i) \\
& =P_{\text {norm }}(i) \frac{P(j) / f\left(d_{i j}\right)}{\sum_{k \neq i} P(k) / f\left(d_{i k}\right),}
\end{aligned}
$$

where $P_{\text {norm }}(\cdot)$ means the normalized population indicating the possibility of originating a trip from the region. Assuming $T$ is the total number of trips, the expected number of trips from region $i$ to $j$ can be concluded as

$$
\begin{aligned}
\left\langle T_{i j}\right\rangle & =T \cdot P(i \rightarrow j) \\
& =\frac{T}{\sum_{k \neq i} P(k) / f\left(d_{i k}\right)} \frac{P_{\text {norm }}(i) P(j)}{f\left(d_{i j}\right)} \\
& =\frac{T}{M(i)} \frac{P_{\text {norm }}(i) P(j)}{f\left(d_{i j}\right)},
\end{aligned}
$$

where $M(i)=\sum_{k \neq i} P(k) / f\left(d_{i k}\right)$.

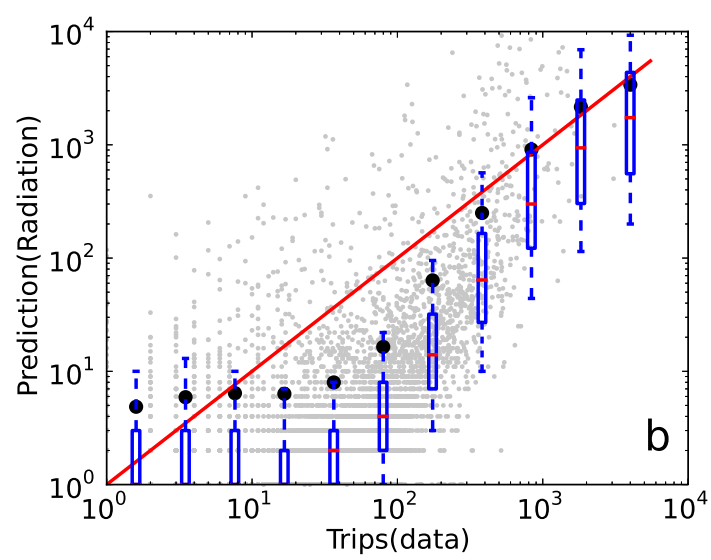

Figure $1 \mid$ The simulations by the radiation model in Beijing. (a) The comparison of distance distributions between actual and simulated trips. (b) The prediction of traffic flows between regions. The grey points show the relationship between actual and predicted flux for ordered pairs of regions. The red line $y=x$ stands for the actual flows equal with predicted ones. The black points are the mean values of predicted flux in the bins. The ends of whisker represent the 9 th and 91 st percentile in the bins. 
As described in the gravity model, the number of trips from region $i$ to $j$ is equal with the one from region $j$ to $i$. However, that is not the case in our model because $M(i)$ and $M(j)$ depend on geographic positions of $i$ and $j$ respectively, which are often not equal to each other. In fact, it is more consistent with actual situations.

After given the actual trips, the parameter of function $f(d)$ in our model can be determined by using the method of Maximum Likelihood Estimation (MLE) (see Methods section). After inspecting the two forms of function $f(d)$ carefully, it is found that the powerlaw form $f(d)=d^{\sigma}$ is much better. The power-law exponents $\sigma$ in our model for the four cities-Beijing, London, Chicago and Los Angelesare $1.601,0.402,1.832$ and 1.805 respectively. By using our model to simulate human travels in the four cities, the relationships between actual and predicted traffic flows are shown in Fig. 2. From the subgraphs, it can be observed that the red lines $y=x$ almost lie between the 9th and the 91st percentiles in all bins except the last bin in Los Angeles, indicating that the model can predict the number of trips between regions accurately. Moreover, the comparisons of distributions of actual and simulated trip lengths are illustrated in Fig. 3. It is discovered that the simulated distributions accord with the actual ones very well in the four cities. The fitted exponential parameters for the simulated distance distributions in the four cities are $0.1828 \pm 0.0001,0.181 \pm 0.0008,0.0903 \pm 0.0017$ and $0.0696 \pm$ 0.0012 respectively, which are very close to the fitted values for actual trip-length distributions as shown in Table S1. In summary, our model with the form of power law can be treated as an appropriate model to predict traffic flows in urban areas.

Analyzing the influence of population distribution. From the model, it is apparent that the spatial population distribution has an important impact on collective human mobility. Taking Beijing as an example, it is investigated that how the geographic population distribution could affect the trip-length distribution. First, considering the population distribution is uniform, individual trips could be predicted by our model with different parameters $\sigma$. As shown in Fig. 4(a), contrary to the actual trip-length distribution, the simulated distance distributions accord to power laws with exponential cutoff very well and decay more slowly. The power-law exponents of the two simulated distributions are -0.716 for $\sigma=1.6$ (green triangles) and -1.100 for $\sigma=2.0$ (red stars), which approach to the analytical results $1-\sigma$ (see SI section II). Second, remaining the distribution of population numbers of cells unchanged, three synthetic population distributions are generated by randomly rearranging the population numbers of cells. In simulations of human trips, the parameter $\sigma$ of our model is the fixed value 1.601, which is the same as the actual one in Beijing. In Fig. 4(b), the simulated distributions are similar to each other and could be described by power-law distributions better. In summary, these demonstrate that not only the distribution of population numbers but also the layout of them could influence the trip-length distribution.

Thus, it is necessary to study the spatial distribution of urban population. Here the relationships between the normalized average density and the distance to urban centers are plotted in Fig. 5 for four cities (see Methods section). From the graph, it can be observed that, for different selected centers of each city, the average urban densities have similar trends. More importantly, the densities for four cities all decay exponentially with the increase of distance to the urban centers. And it is worth noting that the declining slopes are not far from the exponents of exponential estimated from the corresponding distance distributions shown in Table S1.

Assuming the density function $\rho(r)$ is a negative exponential function that depends on the distance $r$ to the center

$$
\rho(r)=C e^{-\lambda r}(\lambda>0,0 \leq r \leq R),
$$

where $\mathrm{C}$ is a constant. The distance distribution $P(d)$ can be derived as

$$
C_{1} d^{1-\sigma} e^{-\lambda d} \leq P(d) \leq C_{2} d^{1-\sigma} e^{-\lambda d},
$$

where $C_{1}$ and $C_{2}$ are constants (see the proof in SI section II). Hence when $d>1 / \lambda$, the exponential section dominates and $P(d)$ begins to decay exponentially.

Then, it is aimed to verify the analytical result through simulating human trips based on our model further. And our model is simulated on grid cells whose size is $80 \times 80$. When fixing the model parameter $\sigma$, as shown in Fig. 6(a), the simulated trip-length distributions all exhibit exponential tails and the parameters of exponential
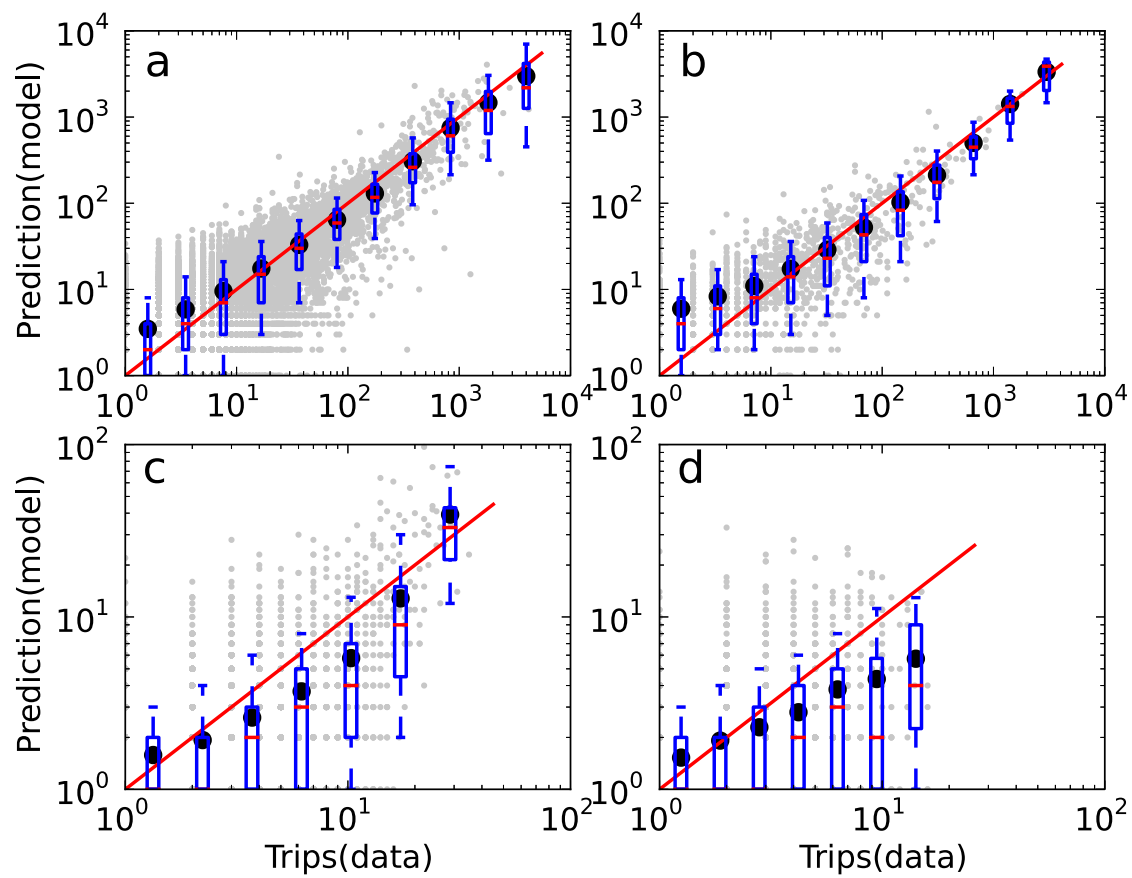

Figure $2 \mid$ The relationships between actual and predicted traffic flux. (a)Beijing. (b)London. (c)Chicago. (d)Los Angeles. 

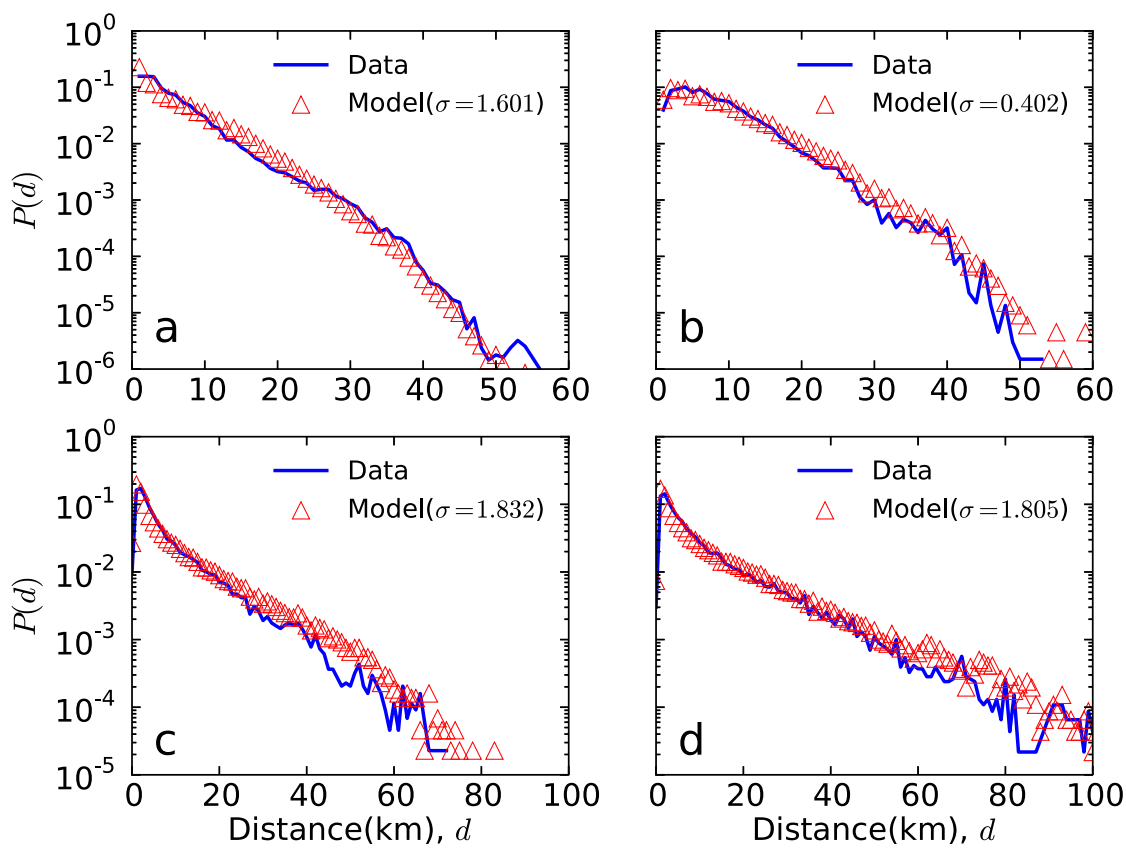

Figure $3 \mid$ The distributions of actual and simulated trip distances in the four cities. The blue solid lines denote the actual traveling distance distributions. The red triangles represent the trip-length distributions simulated by our model. (a)Beijing. (b)London. (c)Chicago. (d)Los Angeles.

distributions approach to the corresponding parameters $\lambda$ of population density distributions. From the Fig. 6(b), the distance distributions have similar rates of exponential decay indicating that the model parameter $\sigma$ has little influence on the exponential tails of distributions when fixing the parameter $\lambda$ of the density function. In conclusion, the result of proof agrees with the simulations very well.

According to the analytical result, it could be explained why the exponential parameters of actual distance distributions are close to the ones of population density distributions in the four cities. Furthermore, in urban areas, the density usually decreases significantly leading to a large exponent $\lambda$, thus a short range of power-law section. Meanwhile, it must be noticed that, since the power of $d$ is often larger than -1 (due to $\sigma<2$ like the four cities in our datasets), $P(d)$ shows such slow power-law decay that it is obviously not a Lévy walk observed in collective human movements in large scale of space. Therefore, it can explain the reason why the distance distribution of human trips in urban areas accords with an exponential distribution much better.

It is worth mentioning that the empirical density distributions in four cities is the same as the Clark's model ${ }^{34}$, which is the most influential model for describing urban population density. Since then, some studies have proposed other mathematical forms for population density. For example, the inverse power function is employed by Smeed ${ }^{35}$. Though some controversies, Parr $^{36}$ suggests that the negative exponential function is more appropriate to model the density in urban areas, while the inverse power function is more appropriate to model the variation of density in the urban fringe and hinterland. Therefore, the phase transition of population density function in different scales of space may be able to explain the different laws emerging in collective human mobility patterns.

\section{Discussion}

In the paper, it is aimed to understand the exponential law of intraurban human mobility at the population level. The four travel datasets in urban areas of cities are analyzed, which further confirm the exponential law. Through considering the travel flows between regions, it is clear that the radiation model is incapable of modeling collective human movements in urban areas. Because of this, a new model is proposed, which can predict traffic flows between regions very well. Based on our model, we discover that the average
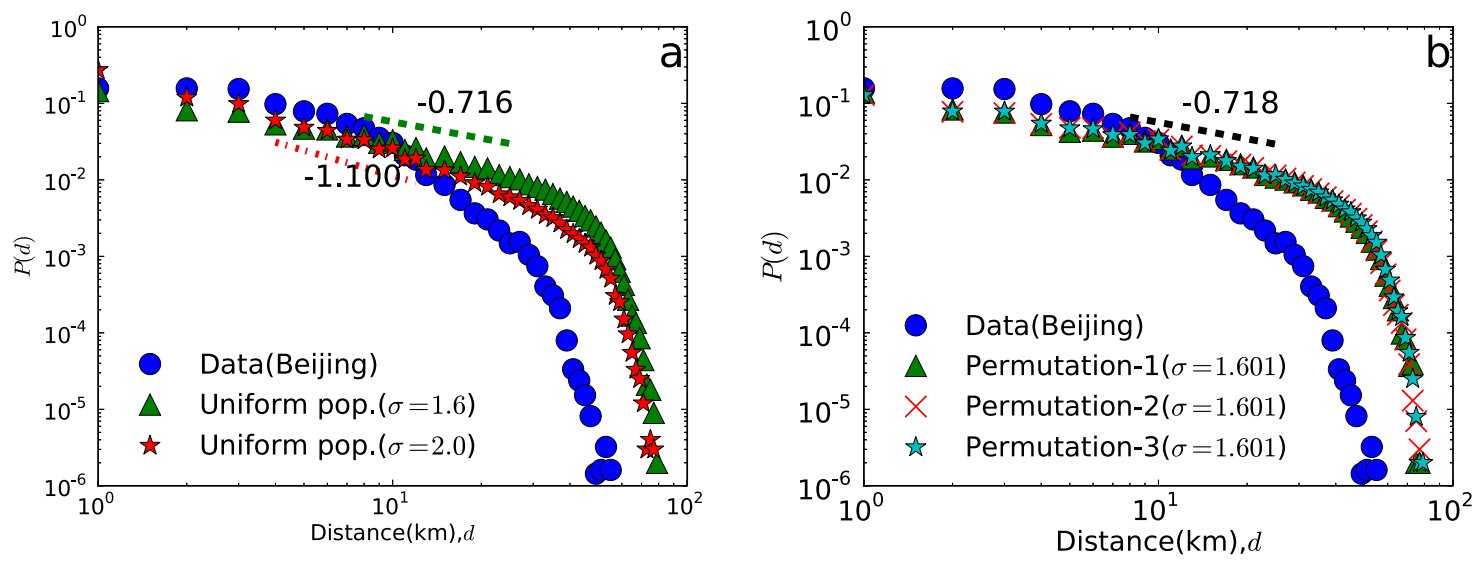

Figure $4 \mid$ The simulated trip-length distributions with different spatial population distributions in Beijing. (a) Uniform population distributions with different model parameters. (b) Randomized permutation of population numbers of cells. 

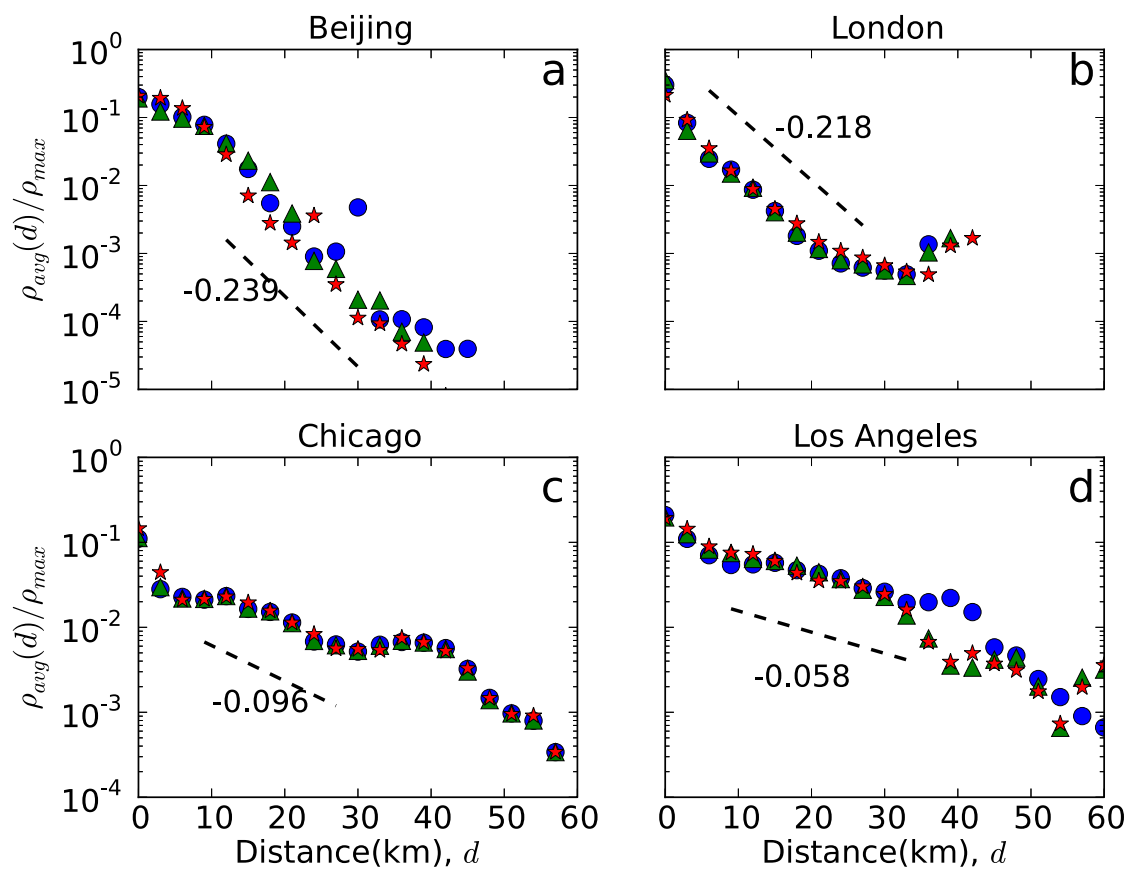

Figure $5 \mid$ The normalized average density versus the distance to urban centers. For each city, three hot regions with high population densities are considered as urban centers. And the blue circles, green triangles and red stars denote the average densities, which are normalized by the maxima of densities, for selected urban centers respectively. The black dashed lines represent the decreasing rates of densities with distance.

population density decreasing exponentially with distance to the urban center ultimately leads to the exponential law of collective human mobility patterns. Moreover, the difference of population distribution in different scales of space might explain the different laws (power-law and exponential) in collective human movements.

In fact, from the exponential law, it is hard to conclude that the trip lengths at the individual level follow a power-law distribution. It must be noted that most empirical studies about human mobility patterns are at the population level, but many models are trying to explore the origin of scaling law at the individual level. Ref. 37 demonstrates that a scale-free distribution of the aggregated movement lengths could also be obtained from individuals with different exponential distributions of movement lengths. A new evidence in human temporal dynamics ${ }^{38}$ shows that although at the aggregated level the intercall durations follow a power-law distribution, but at the individual level they follow a Weibull distribution for the majority. In addition, animal motions inspire the research of human mobility while there are still some controversies ${ }^{39-41}$ on whether animals exhibit Lévy-like behavior. Because of these, the individual mobility patterns should be considered carefully and more comprehensive human travel records are needed for deeply empirical analysis. In the future, we will investigate how the geographical distribution of population can influence the human mobility patterns in large scale of space in detail, to understand the intrinsic reasons of different laws better.

\section{Methods}

Data descriptions. Beijing. The dataset is about the taxis' GPS data generated by over 10 thousand taxis in Beijing, China, during three months ended on Dec. 31st, $2010^{19}$. Based on taxis' locations and statuses of occupation (with passengers or without passengers), trajectories of passengers can be observed. After dividing the urban areas of Beijing (inside the 6th Ring Road) into grid-like cells of 0.01 degree latitude by 0.01 degree longitude, a total of $11,776,743$ trajectories between 3450 cells were extracted.
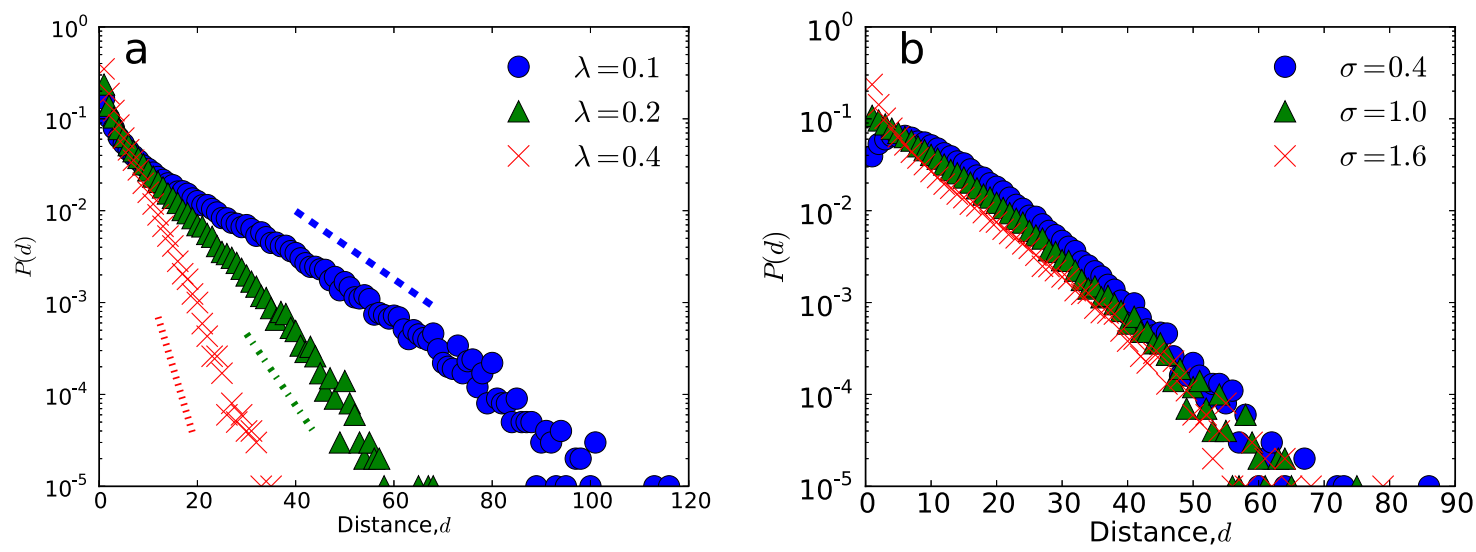

Figure 6 The simulations based on negative exponential distributions of population density. (a) Different population density distributions with the fixed model parameter $\sigma=1.6$. The simulated distance distributions, corresponding to different $\lambda(0.1,0.2$ and 0.4$)$, decay exponentially with slope 0.085 (blue dashed line), 0.180 (green dash-dot line), 0.414 (red dotted line) respectively. (b) Different model parameters with the fixed population density distribution $(\lambda=0.2)$. 
London. The dataset contains about 5\% samples of human trips by Tube in London, which were captured by Oyster cards during a week in November 2009 (available online at http://www.tfl.gov.uk/businessandpartners/syndication/). In the dataset, the stations that a journey started or ended at were recorded. It was noticed that some stations were very close to each other, even less than $200 \mathrm{~m}$. Because of too small area of regions, it could not reflect the regular mobility patterns between regions obviously. After merging some adjacent stations, we obtained 183 voronoi cells based on stations and a total of 667,584 trips between them.

Chicago. The dataset used here comes from the household travel tracker survey in Chicago Metropolitan areas conducted by Chicago Metropolitan Agency for Planning from January 2007 to February 2008 (available online at http://www.cmap. illinois.gov/travel-tracker-survey/). The survey contained various kinds of information about households and travel activities of household members. Among them, the trips occurred in the Cook county were considered which is seen as the urban area of the city according to the population density. Then we extracted a total of 43,881 trips between the 1314 zones which correspond to the census tracts.

Los angeles. The Post-Census Regional Household Travel Survey, sponsored by the Southern California Association of Governments in 2001, was aimed to investigate human travel behavior in the Los Angeles region of California (available online at http://www.scag.ca.gov/travelsurvey/). The region consisted of six counties. In terms of the survey data, it was paid more attention to the movements in the Los Angeles county. As a result, based on the census tracts, the county was divided into 2017 zones and a total of 46,000 tracks were identified between these zones.

MLE for our model. Consider a dataset of intra-urban trips between regions $D=\left\{\left(s e q^{(k)}, r_{o}^{(k)}, r_{d}^{(k)}\right) \mid r_{o}^{(k)}, r_{d}^{(k)} \in S, k=1, \ldots, n\right\}$ in which $n$ is the number of trips, $S$ is the set of regions and each tuple representing a trip consists of the sequence number, the regions of origin and destination. Supposing these trips are independent of each other, the log likelihood function is given by

$$
\begin{aligned}
\log P(D) & =\log \prod_{k=1}^{n} P\left(r_{o}^{(k)} \rightarrow r_{d}^{(k)}\right) \\
& =\sum_{i, j \in S, i \neq j} T_{i j} \log P(i \rightarrow j) .
\end{aligned}
$$

By making use of (4), we obtain

$$
\log P(D)=\sum_{i, j \in S, i \neq j} T_{i j} \log \frac{P_{\text {norm }}(i) P(j) / f\left(d_{i j}\right)}{\sum_{k \neq i} P(k) / f\left(d_{i k}\right)} .
$$

Therefore, the solution for the parameter $\sigma$ of the function $f(d)$ should satisfy

$$
\sigma=\arg \min _{\sigma}(-\log P(D)) .
$$

In this paper, the Nelder-Mead simplex algorithm ${ }^{42}$ is employed to evaluate the parameter $\sigma$ numerically.

Intra-urban population distribution. The spatial distribution of population in a city is often characterized by average population density with the distance to the urban center. As for the dataset of Beijing, the trips are in very fine granularity and there are similar-sized regions (cells) with small area. After selecting the cells with high densities as urban centers, the average densities with distance to centers are calculated easily. But, for the datasets of other three cities, there are coarse granularity of travels and irregular zones. It is not suitable to compute the average density directly. Therefore, assuming that population density in each zone is uniform, the urban area is divided into grid-like cells with size $0.005^{\circ} \times 0.005^{\circ}$. The population density of each cell is regarded as the density of the zone in which the cell lies. Finally, the average densities with distance can be calculated based on these divided grid cells.

1. Rozenfeld, H. D. et al. Laws of population growth. Proc. Natl. Acad. Sci. U.S.A. 105, 18702-18707 (2008).

2. Yuan, J., Zheng, Y. \& Xie, X. Discovering Regions of Different Functions in a City Using Human Mobility and POIs. In Proc. ACM SIGKDD 186-194 (2012).

3. Zheng, Y., Liu, Y., Yuan, J. \& Xie, X. Urban computing with taxicabs. In Proc. Ubicomp 89-98 (2011).

4. Balcan, D. et al. Multiscale mobility networks and the spatial spreading of infectious diseases. Proc. Natl. Acad. Sci. U.S.A. 106, $21484-21489$ (2009)

5. Colizza, V., Barrat, A., Barthelemy, M., Valleron, A.-J. \& Vespignani, A. Modeling the worldwide spread of pandemic influenza: baseline case and containment interventions. PLoS Med. 4, e13 (2007).

6. Wang, B., Cao, L., Suzuki, H. \& Aihara, K. Safety-information-driven human mobility patterns with metapopulation epidemic dynamics. Sci. Rep. 2, 887 (2012).

7. Viboud, C. et al. Synchrony, Waves, and Spatial Hierarchies in the Spread of Influenza. Science 312, 447-451 (2006).

8. Jung, W.-S., Wang, F. \& Stanley, H. E. Gravity model in the Korean highway. Europhys. Lett. 81, 48005 (2008).
9. Goh, S., Lee, K., Park, J. S. \& Choi, M. Y. Modification of the gravity model and application to the metropolitan Seoul subway system. Phys. Rev. E 86, 026102 (2012).

10. Brockmann, D., Hufnagel, L. \& Geisel, T. The scaling laws of human travel. Nature 439, 462-465 (2006).

11. González, M., Hidalgo, C. \& Barabási, A.-L. Understanding individual human mobility patterns. Nature 453, 779-782 (2008).

12. Song, C., Koren, T., Wang, P. \& Barabási, A.-L. Modelling the scaling properties of human mobility. Nat. Phys. 6, 818-823 (2010).

13. Jiang, B., Yin, J. \& Zhao, S. Characterizing the human mobility pattern in a large street network. Phys. Rev. E 80, 1-11 (2009).

14. Viswanathan, G. et al. Levy flight search patterns of wandering albatrosses. Nature 381, 413-415 (1996).

15. Sims, D. W. et al. Scaling laws of marine predator search behaviour. Nature 451, 1098-1102 (2008).

16. Humphries, N. E. et al. Environmental context explains Lévy and Brownian movement patterns of marine predators. Nature 465, 1066-1069 (2010).

17. de Jager, M., Weissing, F. J., Herman, P. M. J., Nolet, B. A. \& van de Koppel, J. Lévy Walks Evolve Through Interaction Between Movement and Environmental Complexity. Science 332, 1551-1553 (2011).

18. Veloso, M., Phithakkitnukoon, S., Bento, C., Fonseca, N. \& Olivier, P. Exploratory Study of Urban Flow using Taxi Traces. In Workshop PURBA (2011).

19. Liang, X., Zheng, X., Lv, W., Zhu, T. \& Xu, K. The scaling of human mobility by taxis is exponential. Physica A 391, 2135-2144 (2012).

20. Peng, C., Jin, X., Wong, K.-C., Shi, M. \& Liò, P. Collective Human Mobility Pattern from Taxi Trips in Urban Area. PLoS ONE 7, e34487 (2012).

21. Bazzani, A., Giorgini, B., Rambaldi, S., Gallotti, R. \& Giovannini, L. Statistical laws in urban mobility from microscopic GPS data in the area of Florence. J. Stat. Mech. 2010, P05001 (2010).

22. Roth, C., Kang, S. M., Batty, M. \& Barthélemy, M. Structure of urban movements: polycentric activity and entangled hierarchical flows. PLoS ONE 6, e15923 (2011).

23. Kang, C., Ma, X., Tong, D. \& Liu, Y. Intra-urban human mobility patterns: An urban morphology perspective. Physica A 391, 1702-1717 (2012).

24. Noulas, A., Scellato, S., Lambiotte, R., Pontil, M. \& Mascolo, C. A tale of many cities: universal patterns in human urban mobility. PLoS ONE 7, e37027 (2012).

25. Yan, X., Han, X., Wang, B. \& Zhou, T. Diversity of individual mobility patterns and emergence of aggregated scaling laws. Sci. Rep. 3, 2678; DOI:10.1038/ srep02678 (2013).

26. Szell, M., Sinatra, R., Petri, G., Thurner, S. \& Latora, V. Understanding mobility in a social petri dish. Sci. Rep. 2, 457 (2012).

27. Han, X., Hao, Q., Wang, B. \& Tao, Z. Origin of the scaling law in human mobility: Hierarchy of traffic systems. Phys. Rev. E 83, 2-6 (2011).

28. Hu, Y., Zhang, J. \& Huan, D. Toward a general understanding of the scaling laws in human and animal mobility. Europhys. Lett. 96, 38006 (2011).

29. Jia, T., Jiang, B., Carling, K., Bolin, M. \& Ban, Y. An empirical study on human mobility and its agent-based modeling. J. Stat. Mech. 2012, P11024 (2012).

30. Barthélemy, M. Spatial networks. Phys. Rep. 499, 1-101 (2011).

31. Kaluza, P., Kölzsch, A., Gastner, M. T. \& Blasius, B. The complex network of global cargo ship movements. J. R. Soc. Interface 7, 1093-1103 (2010).

32. Krings, G., Calabrese, F., Ratti, C. \& Blondel, V. D. Urban gravity: a model for inter-city telecommunication flows. J. Stat. Mech. 2009, L07003 (2009).

33. Simini, F., González, M. C., Maritan, A. \& Baraási, A.-L. A universal model for mobility and migration patterns. Nature 484, 96-100 (2012).

34. Clark, C. Urban Population Densities. J. R. Stat. Soc. (Series A) 114, 490-496 (1951).

35. Smeed, R. J. Road development in urban areas. J. I. Highw. Eng. 10, 5-30 (1963).

36. Parr, J. B. A Population-Density Approach to Regional Spatial Structure. Urban Stud. 22, 289-303 (1985).

37. Petrovskii, S., Mashanova, A. \& Jansen, V. A. A. Variation in individual walking behavior creates the impression of a Lévy flight. Proc. Natl. Acad. Sci. U.S.A. 108, 8704-8707 (2011).

38. Jiang, Z.-Q. et al. Calling patterns in human communication dynamics. Proc. Natl. Acad. Sci. U.S.A. 110, 1600-1605 (2013).

39. Edwards, A. M. et al. Revisiting Lévy flight search patterns of wandering albatrosses, bumblebees and deer. Nature 449, 1044-1048 (2007).

40. Edwards, A. M., Freeman, M. P., Breed, G. a. \& Jonsen, I. D. Incorrect likelihood methods were used to infer scaling laws of marine predator search behaviour. PLoS ONE 7, e45174 (2012)

41. Sims, D. W. \& Humphries, N. E. Levy flight search patterns of marine predators not questioned: a reply to Edwards et al. arXiv: 1210.2288 (2012).

42. Nelder, J. A. \& Mead, R. A Simplex Method for Function Minimization. The Computer Journal 7, 308-313 (1965).

\section{Acknowledgments}

We would like to thank Michael Danziger, Prof. Tao Zhou and Xiao-Yong Yan for their valuable suggestions, which improve this paper greatly. The research was supported by the National High Technology Research and Development Program of China (863 Program) (No. 2012AA011005), the fund of the State Key Laboratory of Software Development Environment (SKLSDE-2011ZX-02). Liang X. and Zhao J. C. thank the Innovation Foundation of BUAA for PhD Graduates(YWF-12-RBYJ-036 and YWF-13-A01-26). 


\section{Author contributions}

X.L., J.Z. and K.X. designed research; X.L. and L.D. performed the experiments, analyzed the data, and prepared the figures. X.L., J.Z. and K.X. wrote the paper.

\section{Additional information}

Supplementary information accompanies this paper at http://www.nature.com/ scientificreports

Competing financial interests: The authors declare no competing financial interests.
How to cite this article: Liang, X., Zhao, J.C., Dong, L. \& Xu, K. Unraveling the origin of exponential law in intra-urban human mobility. Sci. Rep. 3, 2983; DOI:10.1038/srep02983 (2013).

(c) (i) (2) This work is licensed under a Creative Commons AttributionNonCommercial-ShareAlike 3.0 Unported license. To view a copy of this license visit http://creativecommons.org/licenses/by-nc-sa/3.0 